BACTERIOLOGICAL NOMENCLATURT $A N D$ TAXONOMY

\title{
ON THE INADVISABILITY OF SEPARATING THE GENERA MICROCOCCUS AND STAPHYLOCOCCUS
}

\author{
William P. Van Eseltine \\ Department of Veterinary Hygiene \\ School of Veterinary Medicine \\ University of Georgia, Athens, Georgia
}

As pointed out in the International Bulletin of Bacteriological Nomenclature and Taxonomy by the Editorial Board (1954), there seems to have been a tacit assumption by many workers that Micrococcus Cohn 1872 and Staphylococcus Rosenbach $188 \overline{\text { constitute two differentiable genera; but }}$ evidence to support this view appears to be entirely lacking. The attempt, initiated by the Winslows (1908), to separate the more parasitic orange and white staphylococci from the more saprophytic yellow and red micrococci has been criticized by both Hucker (1924a) and Rahn (1929b) and has been largely abandoned. Hucker (1924a), after studying a collection of 316 strains of the micrococcus-staphylococcus group, reported ( $p .72)$ that "no correlations of characters were found that indicated that there were natural subdivisions of this group."

Although aware of the lack of evidence concerning natural subdivisions, Hucker $(1924 b, 1928)$ decided upon an arbitrary separation of the mass-forming cocci into 18 species 1 based primarily on pigmentation, gelatin liquefaction, nitrate reduction, and utilization of ammonium phosphate as sole nitrogen source. With only minor changes, this is the system adopted in the current edition of Bergey's Manual (Breed et al., 1948).

That Hucker's classification is indeed an arbitrary one has been emphasized by Rahn (1929a) who called attention to

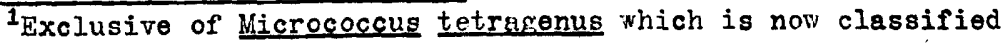
as Gaffkra tetrasena.
} 
the great variability of these cocci (among Hucker's 316 strains only 9 were duplicates) and pointed out the complete parallelism within the group. Rahn's tables and charts show that the various strains of micrococci can be arranged in series, each strain differing from the next by a single property, and with orange, yellow, and white parallels in almost every case. Rahn's evidence is quite convincing, as is his conclusion that, "Any division line is arbitrary whether it be called genus or species division. Really, the entire group of micrococci behaves like one species with an enormous number of different varieties the extremes of which are widely different." It would appear that the micrococcusstaphylococcus group cannot be divided into species (nor genera) on the basis of the characteristics now used in their classification.

Recently it has again been suggested that an attempt be made to separate Staphylococcus from Micrococcus, this time based upon utilization of ammonium phosphate and asparagine as sole sources of nitrogen. In this journal, the Editorial Board (1954) has suggested the same possibility and invited discussion upon this and other points. A reappraisal of Hucker's (1924a) report, the only study to date giving complete results for all strains, reveals that such a separation is unwise.

Hucker's 316 strains vary in Gram-reaction, but nearly all show some tendency to be Gram-positive. In addition, they are all alike in cell form, size, and arrangement; in failure to produce spores or flagella, or to form indole; and in being aerobic to facultative. They vary in the other properties recorded, and a large number of the possiblevarieties are found among the 316 strains. However, none of these is represented by a large enough number of strains to permit much comparison except for the most common type represented by 136 strains all showing the following properties:

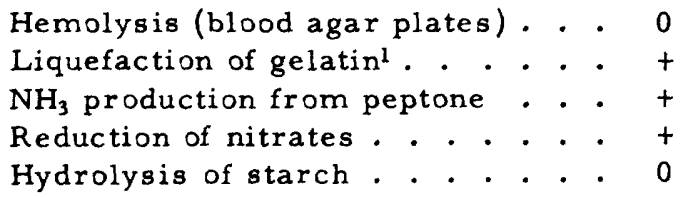

\footnotetext{
${ }^{1}$ Time required for liquefaction, which varied considerably,
} is not stated in Hucker's report. 


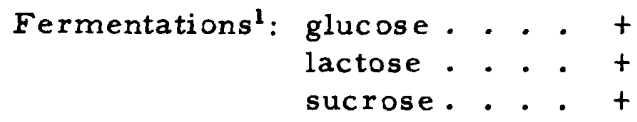

In Table 1 these 136 strains are compared with respect to the remaining properties of pigmentation, reactions in litmus milk, and ability to utilize ammonium phosphate and/or asparagine as sole nitrogen source. It is interesting to note that according to the presentedition of Bergey's Manual, the ammonium phosphate-negative strains would be identified as Micrococcus pyogenes var. aureus (orange), $M$. citeus (yellow) or $M$. pyogenes var. albus (white) provided litmus milk is acidified and curdled. There is no provision for the strains producing other reactions in milk, although most bacteriologists would probably class those producing insufficient acid to curdle, and perhaps those producing no change in milk, as varieties of the same three species. Among the ammonium phosphate-positive strains, those showing an acid proteolysis in milk would be classed as $\mathrm{M}$. caseolyticus regardless of color, while those producing acid only, with no curd, would be $M$. conglomeratus (yellow) or gelatin-liquefying $M$. epidermidis (white). There appears to be no provision for the other ammonium phosphate-positive strains. The full complement of intermediate forms between the "saprophytic" $M$. caseolyticus and the three typically "parasitic" species is noteworthy.

A study of Table 1 reveals scarcely any exceptions to the generalization that for each color of pigment, and for each reaction in litmus milk, there are some strains which can utilize neither ammonium phosphate nor asparagine as sole nitrogen source, others which utilize asparagine only, and still others which can utilize both substances. The only combination which seems not to occur is ability to utilize ammonium phosphate coupled with failure to utilize asparagine. However, this is probably not a "missing link" in the sequence, but rather exactly what one should expect. Micrococci which require no organic nitrogen, but can formall amino acids, proteins, and other nitrogenous cell constituents from ammonium ions, must possess a variety of enzymes (deaminases, transaminases, etc) which can add

\footnotetext{
${ }^{1}$ Ferrentation of $E^{l}$; cerol and mannitol were also studied, but the resulis are nct contained in the publislied recort.
} 


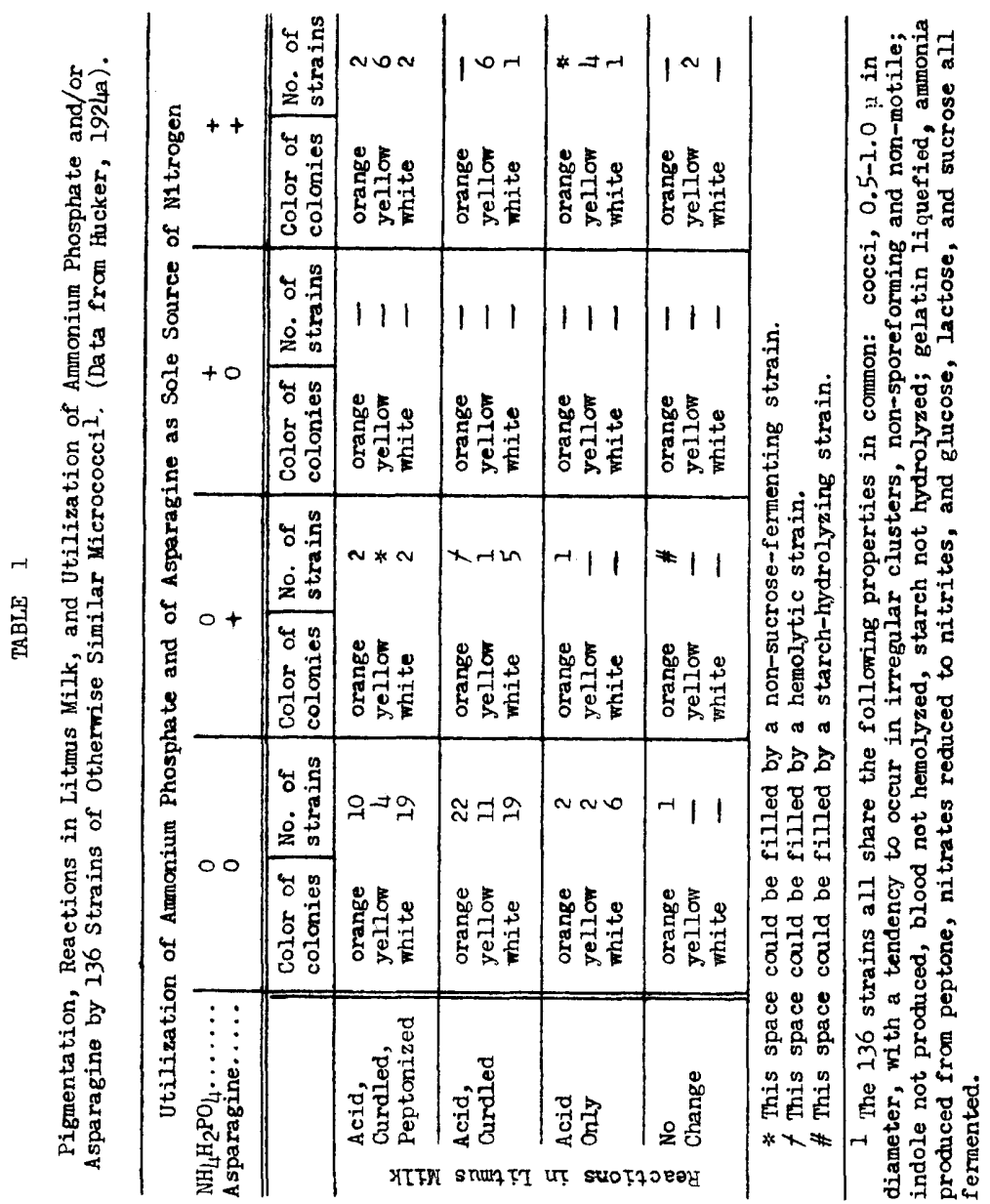


amino groups to carbon chains, remove them for transfer to other molecules, and the like. Doubtless some of these enzymes could remove amino groups from asparagine for transfer to other substances. Thus one would expect strains capable of utilizing ammonium phosphate to be able also to utilize asparagine.

Other strains of the micrococci have lost the ability to synthesize one or more of the amino acids, and consequently cannot grow with ammonium ions as sole nitrogen source. In those few instances where the amino acid which cannot be synthesized is aspartic acid(or an acid which can be formed from aspartic), asparagine will serve as nitrogen source; but with strains unable to synthesize any other amino acid, neither ammonium phosphate nor asparagine can satisfy the deficiency.

With respect to utilization of ammonium phosphate or as paragine, as with the other properties previously considered by Rahn (1929a), the micrococci are extremely variable and appear to form a continuous series including every conceivable combination of characters, and with nogaps which might be utilized for lines of demarcation between species or genera. Separation of genera on the basis of these characters is no more justified at the present time than the old separation based on pigment and habitat. It would only lead to the placing in different genera of otherwise identical organisms.

While utilization of ammonium phosphate coupled with failure to utilize asparagine does not occur a single time among the 136 strains considered in Table 1, this combination is recorded for 11 strains among the 316 used in Hucker's study. Whether these 11 instances represent extremely unusual occurrences, or whether they result from errors in testing or recording results cannot be determined with certainty. That typographical errors can scarcely be avoided in such long and complicated tables as Hucker used is indicated by the fact that four strains are recorded in the tables as producing indole, while the text clearly states that not one of the 316 strains produced indole when tested by four different methods.

At any rate, these 11 instances do not invalidate the argument that organisms utilizing ammonium ions should ordinarily be able to utilize asparagine also, or that the 136 strains of Table 1 show a continuous gradation of synthetic ability. Interesting in this connection is the observation of 
Gladstone (1937) that on primary isolation from suppurative processes, Staphylococcus aureus commonly requires a number of amino acids, but on continual subculture on the usual laboratory media, such nutritive requirements become progressively simpler, until eventually growth can be obtained in inorganic synthetic media containing an ammonium salt and organic carbon. This would seem to indicate that the loss of ability to synthesize one or more amino acids, as postulated above, need not be a complete loss. Rather, the parasitic habitat tends to favor the proliferation of those cells able to utilize most rapidly the preformed amino acids found in the host tissues, while transfer to media lacking amino acids favors the multiplication of the cells capable of most rapid synthesis starting from ammonia.

While the mass-forming cocci are extremely variable with respect to the characters now used in their classification, it is, of course, possible that some more stable criterion may yet be discovered upon which a valid separation could be based. For some time, the ability to coagulate oxalated or citrated plasma has been used successfully as an indication of pathogenicity, and Evans (1948) has shown a high degree of correlation between coagulase production and anaerobic fermentation of mannitol; also between coagulase production and absence of a nutritional requirement for biotin. The difficulties here, as might be expected from the known variability of the group, are that some few of the coagulase negative strains also ferment mannitol anaerobically, and some appear to grow slowly without biotin. Further exploration of these possibilities is indicated, but at best they are likely to prove to be differences between species rather than genera.

It is concluded that on the basis of information now available, the micrococci cannot be considered as constituting more than one genus. Whether this genus should be called Micrococcus or Staphylococcus is debatable, although Micrococcus Cohn 1872 clearly has priority and would appear to be the name of choice provided only that a suitable culture can be found toconform to the description of the type species, Micrococcus luteus (Schroeter) Cohn. In this connection it may be noted that the strain designated as American Type Culture Collection No. 398 cannot serve as type culture if (as stated by Gibson, 1953) it fails to grow in Schroeter's tartrate- $\mathrm{NH}_{3}$-salts medium. There is a c ansiderable differ- 
ence between the ability of an organism to utilize ammonium tartrate as sole source of nitrogen, carbon, and energy, and the ability to grow in ammonium tartrate media only when supplied with an additional source of carbon and energy. A.T.C.C. No. 398 appears to fall within the latter category (Breed, as cited by the Editorial Board, 1954).

\section{SUMMARY AND CONCLUSIONS}

1. Previous workers have shown that on the basis of the characteristics now used in their classification, the organisms of the micrococcus-staphylococcus group cannot be separated into species ( or genera) except in a purely arbitrary and artificial manner.

2. A reappraisal of the only available study giving complete results for all strains shows that with respect ot utilization of ammonium phosphate and asparagine as sole nitrogen sources, these organisms constitute a continuous series which includes every conceivable combination of characters, and shows no gaps which might be utilized for lines of demarcation between species or genera.

3. It is concluded that separation of the genera Micrococcus and Staphylococcus at the present time is unwise.

4. Micrococcus Cohn 1872 would be the correct generic name for these organisms provided only that a type culture can be found which conforms to the original description of the type species, Micrococcus luteus (Schroeter) Cohn.

5. The strain designated as American Type Culture Collection No. 398 cannot serve as type culture if it fails to grow in media containing ammonium tartrate as the sole source of nitrogen, carbon, and energy.

\section{REFERENCES}

Breed, R.S., E.G.D. Murray, and A.P. Hitchens. Bergey's Manual of Determinative Bacteriology, 6th Ed. Baltimore (The Williams and Wilkins Co.) 1948.

Editorial Board. The bacterial generic names Micrococcus and Staphylococcus. Preliminary statement. Int. Bull. Bact. Nom. and Tax. 4(3-4):163-166. 1954 .

Evans, J.B. Studies of staphylococci with special reference to the coagulase-positive types. J. Bact. 55:793-800. 1948 . 
Gibson, T. The status of the generic names Micrococcus and Staphylococcus and of the species name Staphylococcus aureus. Request for an Opinion. File No. 23. Int. Bull. Bact. Nom. and Tax. 3(2-3):129-133, 1953.

Gladstone, G. P. The nutrition of Staphylococcus aureus; nitrogen requirements. Brit. J. Exp. Path. 18:322-333. 1937.

Hucker, G.J. Studies on the Coccaceae. II. A study of the general characters of the micrococci. N.Y. State Agr. Exp. Sta. Tech. Bull. 100. 1924a.

- Studies on the Coccaceae. IV. The classification of the genus Micrococcus Cohn. N.Y. State Agr. Exp. Sta. Tech. Bull. 102.1924b.

- Studies on the Coccaceae. IX. Further studies on the classification of the micrococci. N. Y. State Agr. Exp. Sta. Tech. Bull. 135. 1928.

Rahn, $O$. Contributions to the classification of bacteria. IV. Intermediate forms. Centbl. f. Bakt. Abt. 2, 78:821. 1929 a.

- Contributions to the classification of bacteria. VI. Parasitism as a taxonomic character. VII. Pigmentation as a taxonomic character. Centbl. f. Bakt. Abt. 2, 79: 334-337. $1929 b$.

Winslow, C.E.A., and A.R. Winslow. The Systematic Relationships of the Coccaceae. New York (John Wiley and Sons). 1908. 